\title{
Deux volets fragmentaires \\ du peintre rhénan Barthel Bruyn au château de Peralada
}

\author{
Didier Martens \\ Chargé de cours \\ Université Libre de Bruxelles \\ Faculté de Philosophie et Lettres \\ 50, av. F. Roosevelt (C.P. 175) \\ B-1050 Bruxelles
}

RÉSUMÉ

L'auteur fait connaître deux volets fragmentaires de triptyque dus au peintre rhénan Barthel Bruyn (vers 1493-1555), figure majeure de la peinture colanaise de la Renaissance. Ils sont conservés au château de Peralada (Gérone). Les volets, qui représentent deux familles apparentées et leurs saints patrons, portent des armoiries qui ont été remainées. Dans l'état actuel, elles ressemblent beaucoup à celles d'une ancienne famille patricienne colanaise, les Von Siegen. Le triptyque dont proviennent les fragments de Peralada a dû appartenir à un certain moment à cette famille. C'est vraisemblablement au xix ème siècle qu'il fut démembré, pour être mis au goût du jour. Les volets furent alors séparés du panneau central (disparu), et les saint patrons furent éliminés. Les portraits des donateurs devinrent ainsi des portraits «autonomes», au sens moderne du terme. Les deux volets du triptyque primitif, réduits à l'état de fragment, furent toutefois réunis en un diptyque, de façon à produire un effet d'ensemble «médiéval».

Mots clé:

peinture, Renaissance, Pays-Bas.

Dos fragments del tríptic del pintor Barthel Bruyn de Renània al castell de Perelada

L'autor presenta dos fragments de tríptic del pintor Barthel Bruyn, de Renània (ca. 1493-1555), protagonista de la pintura renaixentista de Colònia. S’han conservat al castell de Perelada (Girona). Els fragments, que representen dues famílies i els seus sants patrons, mostren escuts que han estat alterats. Ara, recorden molt els d'una antiga família patrícia de Colònia, els Von Siegen. El tríptic degué pertànyer a la família en algun moment. Segurament, fou fragmentat al segle XIX per adequarho al gust de l'època. Les ales foren separades del plafó central (desaparegut) i els sants patrons foren eliminats. Els retrats dels donants es convertiren així en retrats "autònoms», en el sentit modern del terme. Les dues ales del tríptic original, reduïdes a fragment, foren unides en un díptic, per fer la sensació d'un conjunt «medieval».

Paraules clau:

pintura, Renaixement, Flandes. 


\section{$I *$}

Au nombre des objets présentés en 1958, à Bruges, à l'exposition L'art flamand dans les collections espagnoles ${ }^{1}$ se trouvaient notamment quatre tableaux appartenant au financier catalan Miquel Mateu (1898-1972)2. A l'exception de la Madone à la vue de Grenade 3 publiée peu avant la Seconde Guerre Mondiale par Don Angulo Iñiguez, qui y voyait l'oeuvre du mystérieux maître brugeois Petrus Christus $\mathrm{II}^{4}$, les autres peintures prêtées par ce collectionneur étaient inconnues des historiens d'art. Depuis lors, deux d'entre elles - le Christ à la colonne de Hans Memling et les volets de l'Annonciation, dus à un peintre de l'entourage d'Isenbrant ${ }^{5}$ - ont été mentionnées à plusieurs reprises dans la littérature scientifique ${ }^{6}$. Elles font aujourd'hui partie du corpus de référence de la peinture des anciens Pays-Bas. En revanche, les volets attribués, dans le catalogue de l'exposition de 1958, à un «Maître brugeois, Xvième siècle» (figure 1) ne paraissent pas avoir retenu l'attention des historiens d'art'. Il est vrai qu'ils n'étaient pas reproduits.

A l'heure actuelle, l'oeuvre, conservée au château-musée de Peralada (prov. de Gérone), se

* C’est un agréable devoir de remercier ici Jaime Barrachina et Ines Padrosa Gorgot, conservateur et bibliothécaire du château de Peralada (prov. de Gérone), pour les aides multiples qu'ils m'ont apportées.

1. L'art flamand dans les collections espagnoles (cat. exp.), Bruges, Groeningemuseum, 1958. L'exposition fut aussi présentée à Madrid. Cf. Arte flamenco en las colecciones españolas (cat. exp.),
Madrid, Sociedad española de Amigos de Arte, 1958.

2. Voir, sur Miquel Mateu i Pla, «Miquel Mateu i Pla, señor de Peralada», dans: La Vanguardia domingo, 21 décembre, 1986, p. 117-132.

3. L'art flamand, op. cit., $\mathrm{n}^{\circ} .35$.

4. Diego Angulo IÑiguez, «La pintura en Grenada y Sevilla hacia 1500 . Un pintor flamenco en présente sous la forme d'un diptyque. Chaque volet mesure hors cadre 60,5 sur $30 \mathrm{~cm}$. Sur le volet gauche, on aperçoit, agenouillés devant un prie-Dieu, un couple et leurs deux enfants, un garçon et une fille. Le mari devait être décédé à l'époque où la peinture fut réalisée, car son épouse porte la robe et la coiffe noires des veuves. Sur le volet droit figure un autre couple agenouillé, plus jeune. L'épouse a également revêtu l'habit des veuves. Le portrait de son mari est donc lui aussi posthume. Le couple eut sept enfants, quatre garçons et trois filles. Derrière ces deux groupes familiaux, on reconnait les restes de deux figures monumentales, habillées de rouge. Il $s$ 'agissait certainement de saints protecteurs. Celui de droite porte une croix (?).

L'état présent de l'oeuvre résulte d'un remaniement tardif. On ne rencontre jamais à la fin du Moyen Age, au nord des Alpes, de diptyques opposant deux groupes symétriques de donateurs en prière, sans que l'objet de leur adoration n'apparaisse dans l'image. A l'origine, le «diptyque Mateu» était en fait un triptyque. Les donateurs occupaient la face intérieure des volets; l'objet de leur adoration figurait sur le panneau central. Au revers des volets, actuellement parquetés, devaient se trouver des peintures qui ont disparu.
Granada», dans: Archivo español de Arte y Arquelogía, 13, 1937, p. 85-90. Voir, en dernier lieu, Matías Díaz PADrón, dans: Splendeurs d'Espagne et les villes belges 1500 1700 , II (cat. exp.), Bruxelles, Palais des Beaux-Arts, 1985, n' ${ }^{\circ}$. C6.

5. L'art flamand, op.cit., $\mathrm{n}^{\mathrm{os}}$. 7, 18 19.

6. Voir, récemment, Dirk DE Vos, Hans Memling. Het volledige oeuvre, Anvers, p. 248; p. 93.
7. L'art flamand, op cit., $\mathrm{n}^{\circ \mathrm{s}}$. 2021.

8. Voir, sur les diptyques avec portraits de donateurs au nord des Alpes, Laura Deborah Gelfland, Fifteenth-Century Netherlandish Devotional Portrait Diptychs: Origins and Function (thèse), Case Western Reserve University, 1994. 


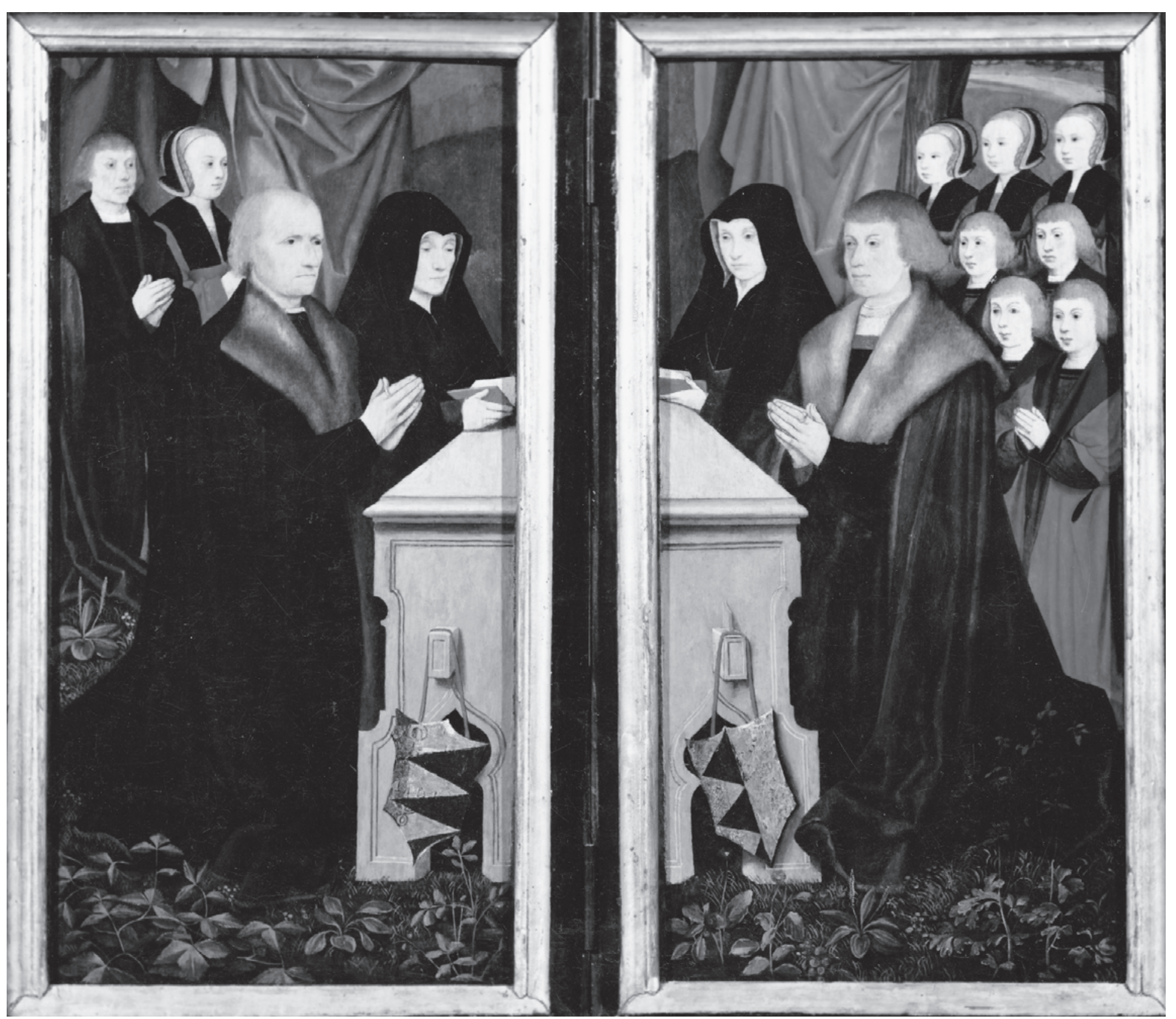

Figure 1.

Attribué ici à Barthel Bruyn l'Ancien «Diptyque Mateu» donateurs et saints patrons. Peralada, collection Carmen Mateu (Photo musée). 
En 1902, le «diptyque», qui faisait partie de la collection Mariano Fuster de Barcelone, figura à une exposition locale d'art ancien. Il était considéré alors comme une oeuvre allemande'. Les organisateurs de l'exposition de 1958 l'attribuèrent, en revanche, à l'école brugeoise. Selon eux, «la disposition des personnages et des prie-Dieu, ainsi que la présence de plantes à l'avant-plan, semblent inspirées du retable de Guillaume Moreel (peint) par Memling» ${ }^{10}$. Les ressemblances existant entre le «diptyque Mateu» et le fameux retable memlinguien (figure 2$)^{11}$ sont toutefois superficielles. La formule consistant à représenter les donateurs d'un triptyque agenouillés à l'air libre devant un prie-Dieu est à ce point fréquente dans la peinture des Xvème et Xvième siècles qu'elle ne saurait constituer un indice en faveur d'une quelconque attribution.

\section{II}

Il convient de retourner à la première attribution. Les deux volets sont en effet dus à Barthel Bruyn dit «l'Ancien» (vers 1493-1555), peintre natif de Wesel qui, après un probable séjour à Kalkar dans l'atelier de Jan Joest, s'installa à Cologne au cours de la deuxième décennie du xvième siècle. Rapidement submergé de commandes prestigieuses, il travailla non seulement pour les nombreuses églises de la métropole rhénane, mais aussi pour le chapitre noble d'Essen et pour la collégiale Saint-Victor de Xanten. Dans le même temps, il développa une importante activité de portraitiste, introduisant à Cologne la mode du portrait de couple sur panneaux autonomes.

Artiste conservateur tout en cherchant à être «de son temps», Barthel Bruyn sut rencontrer les aspirations esthétiques du patriciat colonais. Celuici, resté fidèle à l'ancienne foi alors que la majorité des villes de l'Empire adoptait la Réforme, ne pouvait qu'apprécier un peintre dont l'art préservait les traditions de la peinture rhénane de la fin du Moyen Age, tout en y intégrant certains éléments de la Renaissance italienne. Ces concessions superficielles au goût nouveau apportaient en effet à un style fondamentalement archaique —et aux conceptions religieuses qu'il véhiculait- une sorte de légitimité historique. $\mathrm{Ne}$ faisaient-elles pas apparaître, en plein Xvième siècle, la tradition artistique septentrionale issue du Moyen Age - mais aussi le catholicisme sous sa forme pré-tridentine- comme une tradition toujours vivace, puisque capable de se renouveler?

En 1964, Horst-Johs Tümmers attribuait à Barthel Bruyn 190 retables, volets de retables ou peintures religieuses autonomes, ainsi qu'une fresque ${ }^{12}$. S'y ajoutent les 115 portraits répertoriés en 1965 par Hildegard Westhoff-Krummacher ${ }^{13}$. Depuis lors, le catalogue du peintre a été enrichi de plusieurs inédits ${ }^{14}$. On conserve aujourd'hui plus d'oeuvres de Barthel Bruyn que de n'importe lequel de ses contemporains actifs dans les anciens PaysBas. La chose est due, pour une bonne part, au fait que Cologne échappa au phénomène iconoclaste. En outre, la relative médiocrité de la scène artistique rhénane du Xvilème siècle a sans doute aussi contribué, de manière indirecte, à la remarquable survie de l'art local des époques précédentes. Il n'y eut à Cologne, à l'époque de la Contre-Réforme, aucun «grand maître» susceptible de créer un véritable engouement des élites sociales pour l'art contemporain. Alors que dans les anciens Pays-Bas, l'impact produit par le style rubénien eut pour conséquence que les oeuvres des «Primitifs» furent remplacées de manière systématique par des peintures «modernes», ce qui entraîna de nombreuses pertes, à Cologne, on constate que, bien souvent, les retables gothiques demeurèrent sur les autels pour lesquels ils avaient été conçus, et ce jusqu'à la sécularisation de 1802.

Les physionomies reproduites sur le «diptyque Mateu» portent clairement l'empreinte de Barthel Bruyn et de son répertoire formel. Le personnage masculin âgé qui figure sur le volet gauche peut être comparé à l'un des donateurs du retable de Xanten ${ }^{15}$. L'épouse du volet droit semble une soeur jumelle de la donatrice et de la sainte Catherine figurant sur un volet de Nuremberg ${ }^{16}$. Ces comparaisons suggèrent que le «diptyque Mateu» a dû voir le jour vers 1525-1535. Il s'agirait donc d'une oeuvre de la maturité du peintre.

Des armoiries sont suspendues aux deux prieDieu (figure 3). Sur le volet gauche, l'écu est «partiémanché de six pièces d'or et de sable»; sur le volet droit, il est "parti, à dextre parti-émanché de six pièces d'or et de sable», à senestre d'or au lion rampant». Le lion, que l'on aperçoit bien sur les photographies anciennes ${ }^{17}$, est en partie effacé à l'heure actuelle. Manfred Huiskes (Cologne, Historisches Archiv) a attiré l'attention sur les analogies existant entre les armoiries représentées sur le volet gauche et celles des Von Siegen ${ }^{18}$. L'écu de cette importante famille colonaise est, selon la tradition, "parti-émanché de six pièces d'azur et d'or» ${ }^{19}$.

Les armoiries du «diptyque Mateu» sont sans doute celles des Von Siegen. L'inversion des couleurs -l'or a changé de côté- est un phénomène largement répandu dans l'héraldique ancienne et ne saurait ébranler l'identification proposée. Quant au noir visible sur les écussons du «diptyque Mateu», il résulte sans doute d'une altération du pigment bleu. Celle-ci s'observe souvent dans la peinture médiévale et peut occasionner, sur un blason, la transformation de l'azur en sable. Il est fort douteux, toutefois, que 
9. Catálogo general de la Exposición de Arte antiguo, Barcelone Junta municipal de Museos y Bellas Artes, 1902, nº 371 [ Tabla Díptico. "Un voto de familia" Época gótica. Escuela alemana. Siglo XV a principios del XVI ...»].

10. L'art flamand, op. cit., p. 48.

11. Max J.Friedländer, Early Netherlandish Painting, VI, 1 Hans Memling and Gerard David, Leyde/Bruxelles, 1971, n 12.

12. Horst-Johs Tümmers, Die Altarbilder des Älteren Bartholomaus Bruyn. Mit einem kritischen Katalog, Cologne, 1964.

13. Hildegard WeSTHOFF-KRUMMACHER, Barthel Bruyn der Ältere als Bildnismaler (= Kunstwissenschaftliche Studien, 35), Munich, 1965.

14. Voir, pour la littérature récente consacrée à ce peintre, Didier MARTENS, «Un soi-disant portrait de prédicant au Musée de la Ville de Bruxelles», dans: Revue des Archéologues et Historiens d'Art de Louvain, 25, 1992, p. 90-92, note 16 . Voir aussi, depuis lors idem, «Jeu de rôle et "Imitatic Christi": le "Saint Jérôme pénitent" de Barthel Bruyn du musée de la Chartreuse de Douai», dans: Revue du Nord. Histoire, 77, 1995, p. 483-509; idem, "Conserve-t-on vraiment une oeuvre du peintre- abbé liégeois Jean Peecks?», dans Mélanges Pierre Colman $(=A r t \mathcal{E}$ Fact, 15), Liège, 1996, p. 70-74.

Plusieurs oeuvres méconnues de l'entourage de Barthel Bruyn ont été récemment reproduites et commentées. Voir Wolfgang Been, Deutsche Malerei um 1260 bis 1550 im Hessischen Landesmuseum Darmstadt (= Kataloge des Hessischen Landesmuseums,

15), Darmstadt, 1990 , ns 28,30 $36,38,40$.

15. Tümmers, op. cit., ${ }^{\circ}$ A96, fig. p. 192, en bas à gauche

16. Ibidem, nº. A69.

17. Voir les illustrations publiées dans Pèl i Ploma. Periòdic mensual amb dibuixos (Barcelone), $1903,4, \mathrm{n}^{\circ} 92$ (avril) et les photos ACL, Bruxelles, $n^{\circ}$ B174.444 (1958) et Mas, Barcelone, Caja 12 $\mathrm{n}^{\circ} \mathrm{B}-1789$.

18. Lettre à l'auteur, 5 février 1991.

19. Rietstap, Armorial général, II, $1872^{2}$. Voir, sur cette famille, Herbert M. Schleicher, Die genealogisch-heraldische Sammlung des Kanonikus Joh. Gabriel Von der Ketten in Köln Cologne, 1986, IV, p. 529-533.
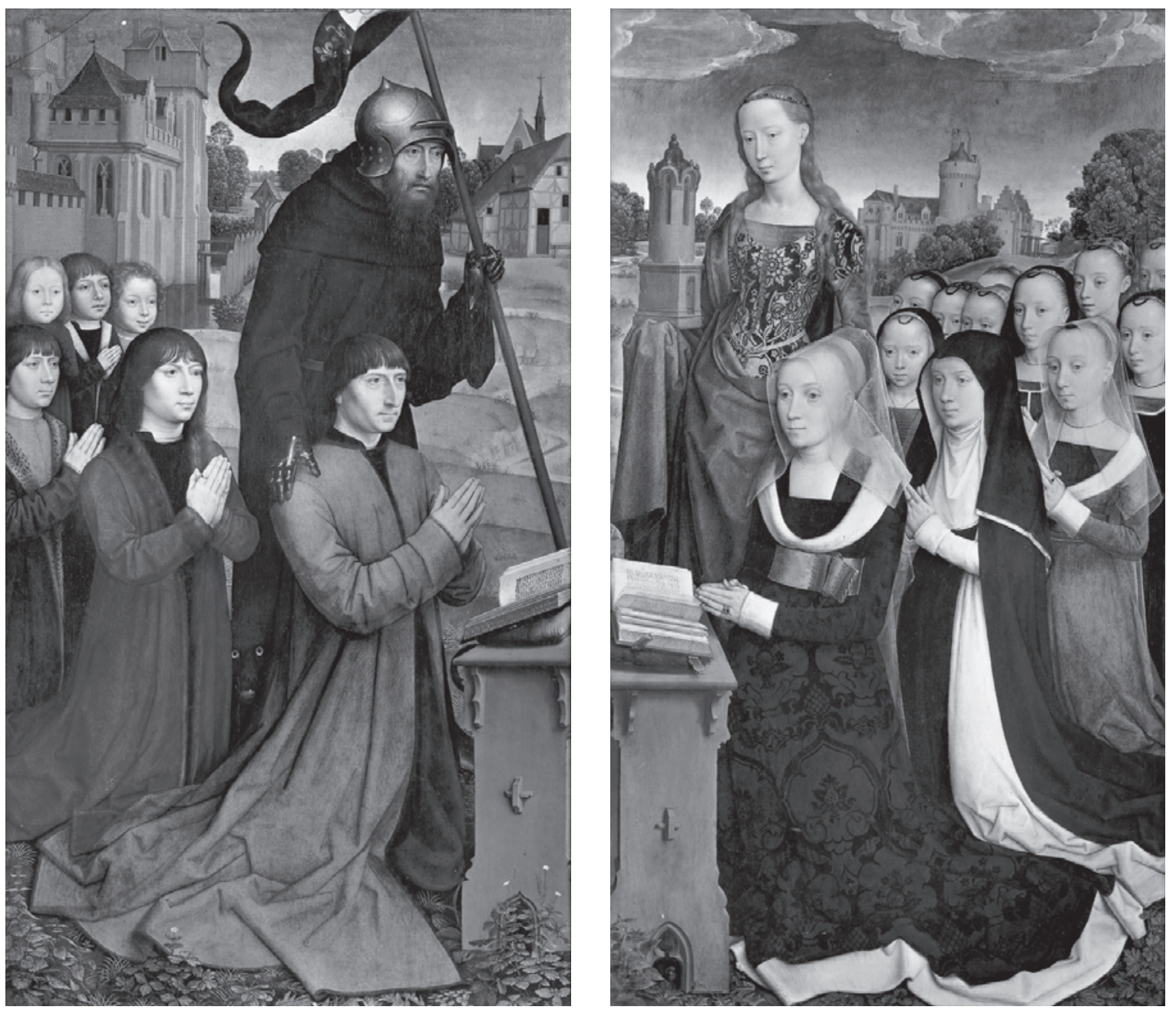

Figure 2.

Hans Memling. Triptyque Moreel, volets avec donateurs et saints patrons. Bruges, Groeningemuseum (Photo ACL, Bruxelles).
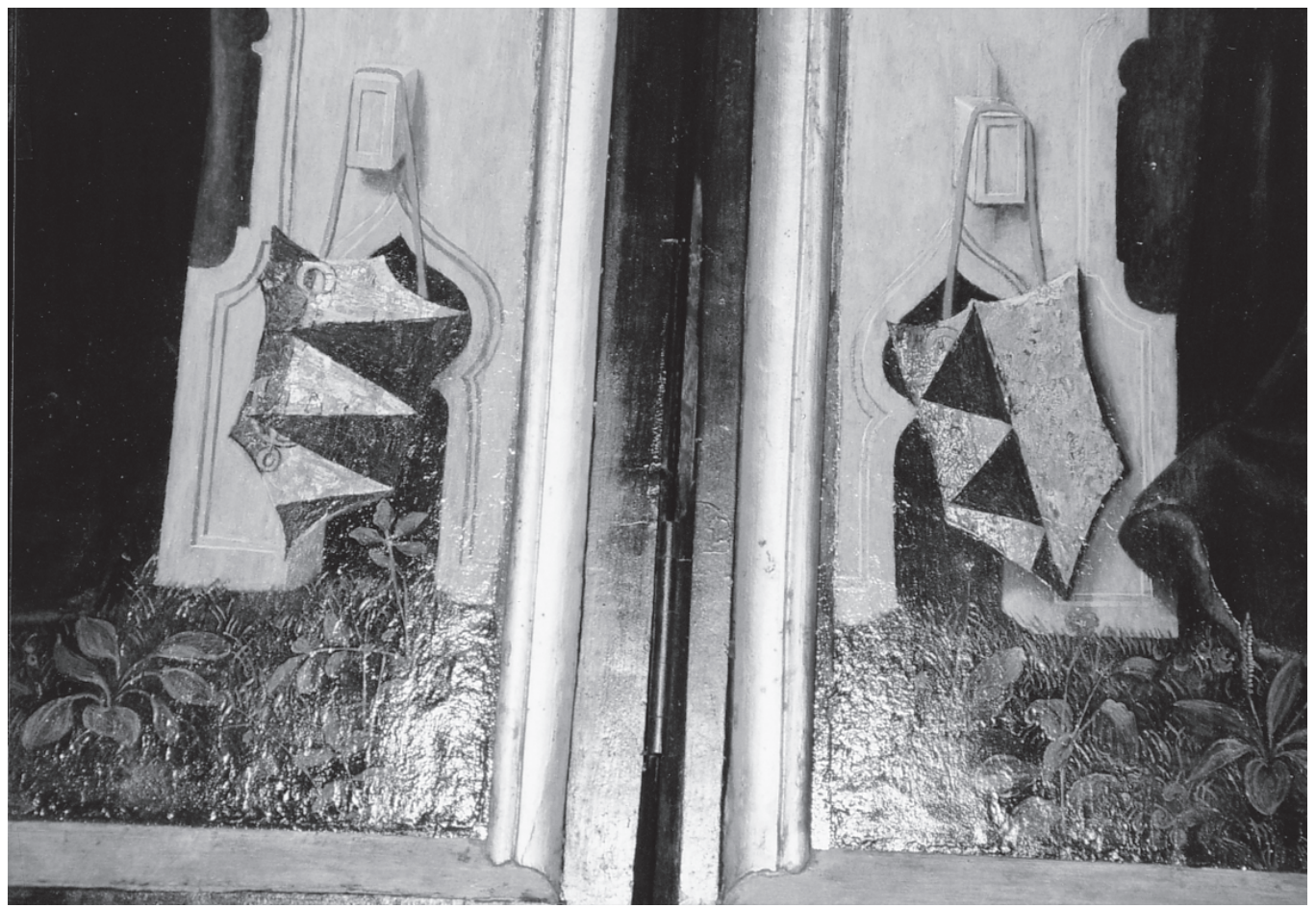

Figure 3

Attribué ici à Barthel Bruyn l'Ancien. Détail de la figure 1. 


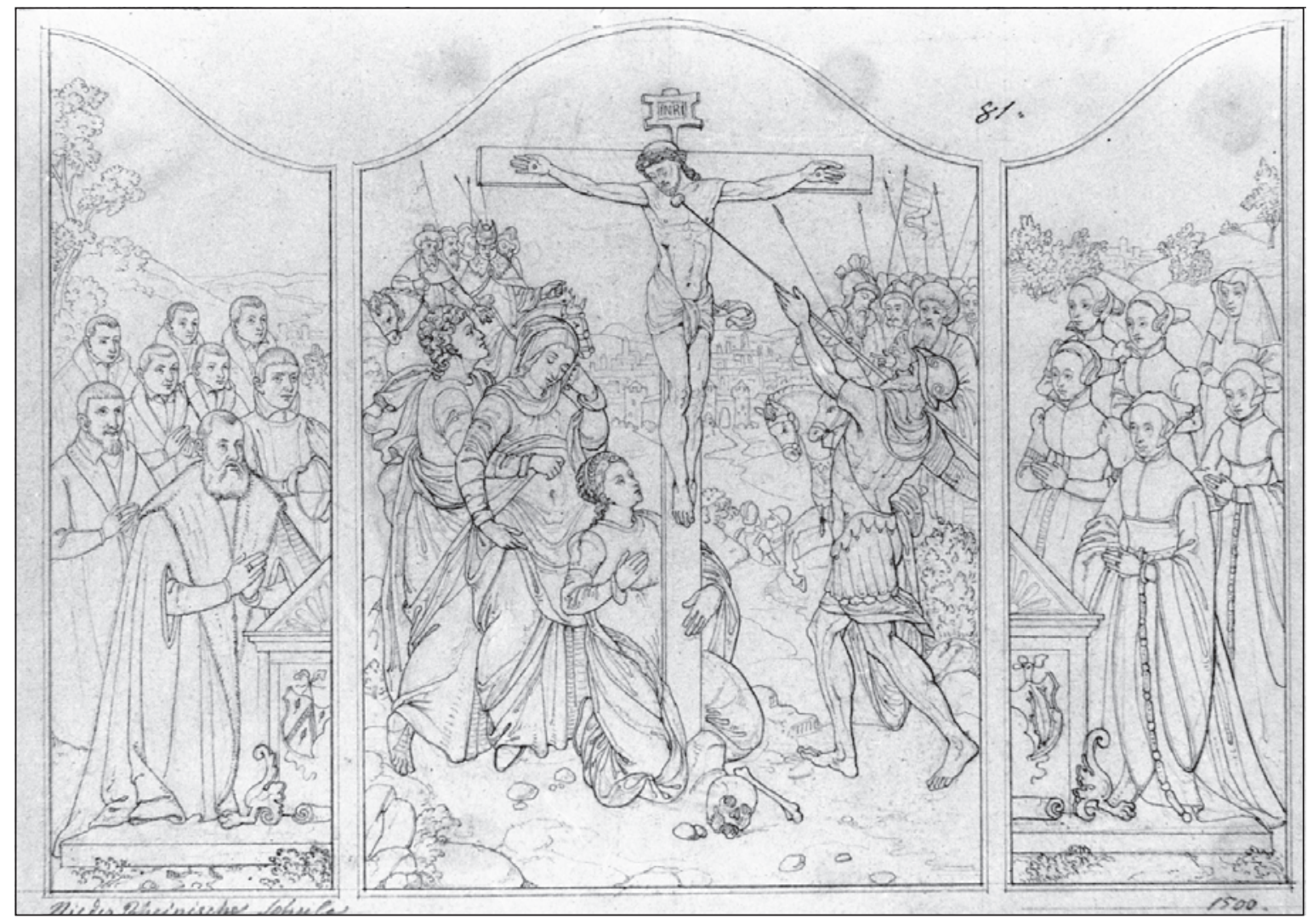

Figure 4.

Barthel Bruyn l'Ancien ou Barthel Bruyn le Jeune (?). Triptyque, Crucifixion avec donateurs.

Anciennement à Cologne, collection Johann Peter Weyer (dessin).

les commanditaires du «diptyque Mateu» aient effectivement été des Von Siegen. On découvre en effet, sous les deux blasons, les restes d'armoiries plus anciennes comportant de chaque côté une ancre d'or brochant sur un fond parti sable et argent. Ce sont certainement les armoiries des personnes qui ont commandé le triptyque et s'y sont fait représenter. L'emblème des Von Siegen fut apposé dans un second temps sur l'oeuvre, sans doute à une date tardive, après que le triptyque eut changé de mains.

\section{III}

La manière dont le peintre a disposé dans l'image les prie-Dieu des donateurs mérite l'attention. Les côtés sont parallèles au plan du tableau, la partie antérieure du meuble et le côté visible sont interrompus par le cadre. Cet agencement des prieDieu dans le champ de l'image produit un effet paradoxal. D'une part, il renforce les suggestions de «présence», puisqu'il fait coïncider l'un des côtés du meuble, comme «engagé» dans le cadre, avec la surface matérielle du volet. Le prie-Dieu représenté tend, de ce fait, à acquérir en partie le caractère de réalité première non-médiée du panneau de bois sur lequel il est peint. D'autre part, le rendu du prie-Dieu sous la forme d'une configuration géométrique élémentaire -un sorte de rectangle surmonté d'un triangle- lui enlève une part de réalité, d'autant plus que seul un fragment de l'objet est visible. Au lieu d'un meuble, le spectateur peut voir aussi, dans les prie-Dieu du «diptyque Mateu», des formes géométriques qui se répondent, des surfaces ornementales.

Semblable «mise en image» du prie-Dieu est caractéristique de Barthel Bruyn et de son entourage. Chaque fois que le maître de Wesel a représenté des donateurs agenouillés devant un prie-Dieu sur les volets d'un triptyque, il a procédé de cette manière.

En témoignent le triptyque fragmentaire de Peter von Clapis partagé entre Cologne et Aixla-Chapelle, ainsi que celui de la famille Von Siegen aujourd'hui à Nuremberg (on aperçoit sur ce triptyque les mêmes armes que sur le volet gauche du «diptyque Mateu» ${ }^{20}$. On signalera aussi les volets colonais datés 1564 d'une Adoration des Mages anversoise vendue chez Lempertz dans les années $70^{21}$, les volets colonais conservés au château du Wawel à Cracovie ${ }^{22}$ et un triptyque avec donateurs jadis dans la collection Weyer de Cologne ${ }^{23}$. Il s'agit, dans ce dernier cas, soit d'une oeuvre tardive de Barthel 
Bruyn, soit d'une oeuvre de Barthel le Jeune. A l'heure actuelle, elle n'est plus connue que par un dessin (figure 4).

Dans le triptyque Moreel, Hans Memling a représenté les prie-Dieu des donateurs d'une autre manière que Barthel Bruyn et ses imitateurs. Même s'il a, lui aussi, disposé les côtés des deux meubles parallèlement au plan de l'image, le côté tourné vers le spectateur apparaît dans sa totalité et n'est pas tangent au cadre. En outre, les prie-Dieux ne sont pas agencés de manière symétrique. Les suggestions illusionnistes contenues dans ces meubles sont, chez Memling, beaucoup plus discrètes que chez Barthel Bruyn, du fait de l'affaiblissement du lien avec le cadre. Quant aux suggestions ornementales, elles ont purement et simplement disparu. Memling a, au contraire, voulu donner des deux meubles une image représentative et mettre en évidence leur fonction, en plaçant le livre de prières bien en vue sur la tablette. Le spectateur voit de ce fait l'objet «prie-Dieu», plus que sa forme.

\section{IV}

Suite à la sécularisation des biens ecclésiastiques en 1802, les innombrables peintures que conservaient les sanctuaires, chapelles et couvents de Cologne furent mises sur le marché. L'objet sacré se transforma en objet de collection. Or, le XIXème siècle ne goûtait pas outre mesure les représentations constituées de panneaux multiples dont le Moyen Age occidental avait été si friand. Il n'aimait guère plus son imagerie souvent hybride, associant le portrait d'un particulier à l'effigie d'un saint et à l'illustration d'un épisode biblique. En dépit de la valorisation inconditionnelle du Moyen Age, l'époque de Friedrich Von Schlegel et des frères Boisserée continuait à préférer le tableau rectangulaire isolé, dont l'usage, au nord des Alpes, se généralisa au Xviı̀̀me siècle, et demeurait attachée à la compartimentation de la thématique picturale en genres indépendants: portrait, peinture d'histoire, paysage, nature morte. Dans ces conditions, on comprend que les triptyques colonais de la fin du Moyen Age eurent à subir diverses altérations ${ }^{24}$.

Restaurateurs et antiquaires mirent au goût du jour ces oeuvres d'un autre âge. C'est ainsi que les volets avec donateurs et saints patrons furent souvent séparés de la scène biblique représentée sur le panneau central. En outre, on scia parfois les volets dans le sens de la largeur, de manière à «libérer» les portraits des commanditaires de l'effigie de leurs saints patrons. D'un «donateur présenté par son saint patron», on fit un «portrait» autonome et un «saint à mi-corps». Il arriva même que le fond du «portrait» obtenu de la sorte fut

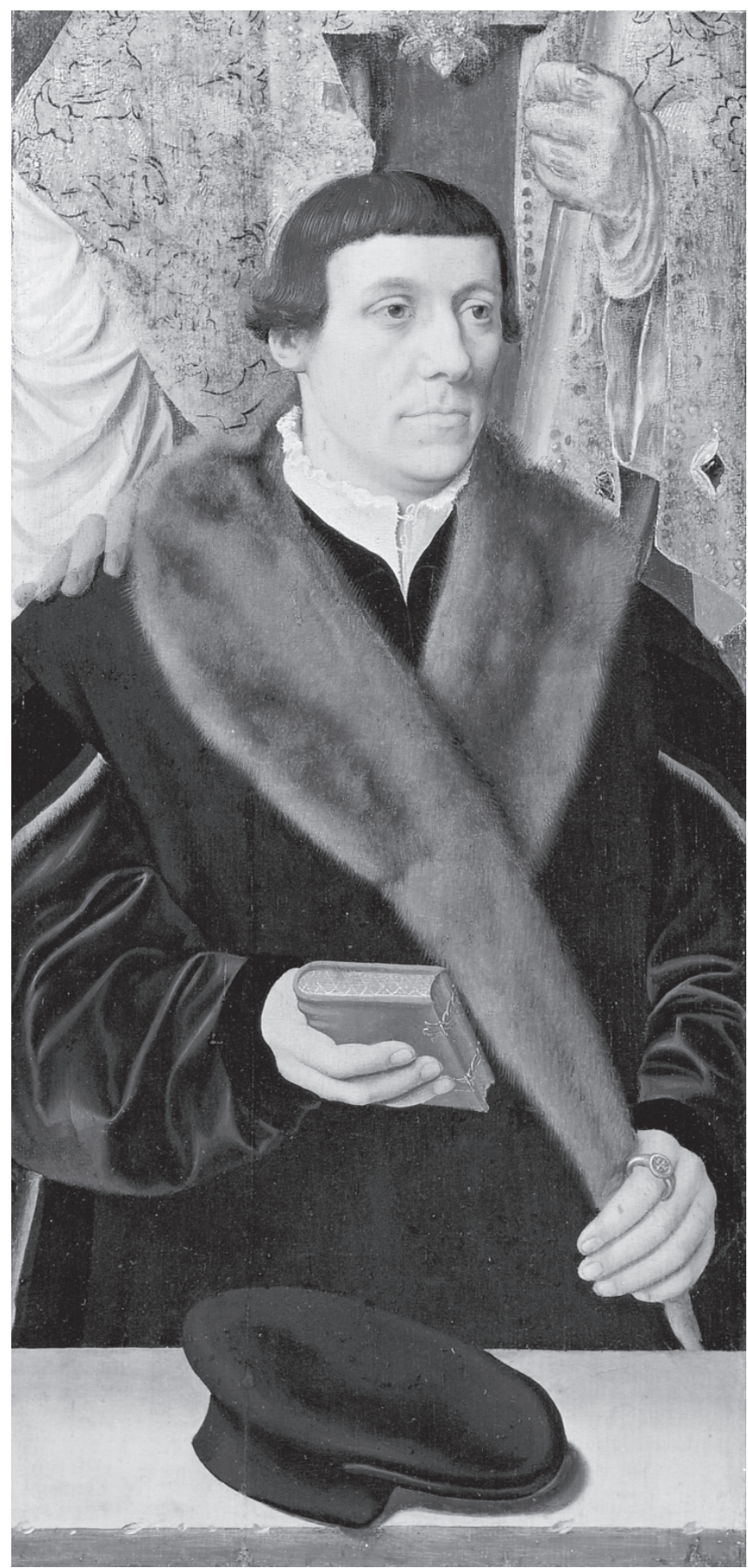

Figure 5.

Barthel Bruyn l'Ancien. Triptyque, volet gauche avec donateur et saint patron. Cologne, Wallraf-Richartz-Museum (Photo Rheinisches Bildarchiv, Cologne).

20. Tümmers, op. cit., $n^{\circ}$ s A75-76; A142.

21. Alte Kunst (cat. de vente), Cologne, Kunsthaus Math. Lempertz, 15-22 nov. 1976, nº 502.

22. Tümmers, op. cit., n ${ }^{\circ}$ s C54-55; Jerzy SzABLOWSKI, Les collections $d u$ Château royal du Wawel, Varsovie, 1975, fig. 81- 82.

23. Host VeY, Johann Peter Weyer. Seine Gemäldesammlung und seine Kunstliebe, dans: WallrafRichartz-Jabrbuch, 28, 1966, p. $211, \mathrm{n}^{\circ} 66$.
24. Les retables italiens du Moyen Age subirent un sort semblable, pour des raisons analogues, ainsi que le rappelle Enrico Castelnuovo dans son introduction à André Chastel, La pala ou le retable italien des origines à 1500 , Paris, 1993, p. 14-15 ( $\mathrm{La}$ fragmentation dont ces oeuvres ont été victimes, généralement entre le xvinìme et le xixème siècle, à l'époque où naquirent l'intérêt pour les Primitifs et la constitution des premières collections orientées en ce sens, fut la conséquence du changement de leur fonction - d'objets de culte, ils devinrent objets de collectionet d'un intérêt passionné mais unilatéral pour la peinture que l'on appréhendait non pas en fonction de l'époque où les oeuvres avaient été créées et placées sur les autels mais de celle où on les avait démontées, dépecées, mutilées, avant de les introduire dans des musées ou des collections»). 
